Original Article Journal of Epilepsy Research pISSN 2233-6249 / elSSN 2233-6257

Received June 14, 2021

Revised August 21, 2021

Accepted September 3, 2021

Corresponding author:

Praveen Sharma, DM

Department of Neurology, King George

Medical University, Shahmina Road,

Chowk, Lucknow, India

Tel. +91-738-8111289

Fax. +91-522-2258852

E-mail; pspgimer@gmail.com

\section{Predictors of Seizure Recurrence in Solitary Calcified Neurocysticercosis in Relation to Computed Tomography Scan: Prospective Observational Study}

\author{
Shinu Singla, MD', Ravindra K Garg, DM¹, Rajesh Verma, DM', Hardeep S Malhotra, DM', \\ Imran Rizvi, DM ${ }^{1}$, Neeraj Kumar, DM ${ }^{1}$, Ravi Uniyal, DM ${ }^{1}$, Shweta Pandey, DM ${ }^{1}$, \\ Anit Parihar, $\mathrm{MD}^{2}$, Praveen Sharma, $\mathrm{DM}^{1}$ \\ ${ }^{1}$ Department of Neurology, ${ }^{2}$ Department of Radiodiagnosis, King George Medical University, Lucknow, India
}

Background and Purpose: Solitary calcified neurocysticercosis (NCC) on the computed tomography (CT) scan of brain in patients of epilepsy is common finding in endemic regions. Factors causing seizures in such cases are debatable. Immature calcification may be the causative factor for seizure recurrence. Thus, we aimed to study predictors of seizure recurrence specific to morphological characteristics on CT scan.

Methods: Patients with solitary calcified NCC on CT scan brain and active seizures were prospectively included. The protocol included clinical evaluation, contrast-enhanced CT scan of the brain, and electroencephalogram (EEG) at baseline and 9th month of 1-year follow-up in all patients. Seizure recurrence after 1 week of enrolment was recorded.

Results: One hundred twenty patients with a mean age of $23.33 \pm 12.81$ years were included with a final follow-up of 109 patients and 35 patients had seizure recurrence. On univariate analysis, seizure frequency of more than 1 episode/month ( $45.7 \%$ vs. $25.7 \%, p=0.037$; odds ratio [OR], 2.06 ; $95 \%$ confidence interval [Cl], 1.05-5.68), perilesional edema on CT head (45\% vs. $10.8 \%, p<0.001$; OR, 6.95; $95 \% \mathrm{Cl}, 2.58-18.7)$, lower density $(\mathrm{HU})$ of lesion on CT head $(139.85 \pm 76.54$ vs. $204.67 \pm 135.9 \mathrm{HU}$ $p=0.009)$ and abnormal EEG at presentation ( $p<0.001$; OR, 18.25; 95\% Cl, 2.15-155.13) were significantly associated with seizure recurrence. On multivariate analysis, presence of perilesional edema on CT head ( $p=0.001$; OR, 6.854; 95\% Cl, 2.26-20.77), density of lesion on CT $(H U)(p=0.036$; OR, 0.995; $95 \% \mathrm{Cl}, 0.99-1)$ and abnormal EEG ( $p=0.029$; OR, $12.125 ; 95 \% \mathrm{Cl}, 1.29-113.74)$ were independently associated with seizure recurrence.

Conclusions: The presence of perilesional edema, HU of calcification on CT brain, and abnormal EEG suggest an increased risk of seizure recurrence in patients of epilepsy with solitary calcified NCC. $(2021 ; 11: 120-126)$

Key words: Neurocysticercosis, Epilepsy, Cysticercus, Computed tomography, Cerebral calcification, Electroencephalogram

\section{Introduction}

Neurocysticercosis (NCC) accounts for $29 \%$ of epilepsy world-wide. ${ }^{1,2}$ Calcified granuloma is the final stage of the parenchymal cysticercus larva. It happens to be the most common radiological finding in the endemic area (10-20\% of the endemic population). ${ }^{3}$ Previously, calcified NCC was supposed to be inert, but recent studies suggest that this calcified granuloma can be epileptogenic. Roughly one-third of the patients with calcified NCC have seizures ${ }^{4}$ and up to $34 \%$ of cases can show seizure recurrence. ${ }^{5}$ The factors responsible for epilepsy in the case of a calcified granuloma are debatable; however, status epilepticus at presentation, serial seizures, perilesional edema, perilesional gliosis, and larger size of the lesion have been suggested to be predictors of seizure recurrence. ${ }^{5,6}$ Blood-brain barrier dysfunction and hippocampal sclerosis secondary to calcified granuloma may be responsible for seizure recurrence, too. ${ }^{3,78}$ Patients of calcified NCC with seizures are usually controlled with anti-epileptic drugs, but a longer duration of treatment may be required due to seizure recurrence. ${ }^{7,9,10}$ Rarely, calcified NCC 
can be the cause of drug-resistant and surgically remediable epilepsy with better outcomes after surgery. ${ }^{11}$

We hypothesized that solitary calcified granuloma with less density of calcification (immature calcification) has a greater propensity to cause a recurrent seizure. Therefore, we planned to study it in detail like quantifying the density of calcified NCC on computed tomography (CT) scan and correlation with seizure recurrence in addition to other morphological characteristics of the calcified granuloma. Clinical and electroencephalographic features were also studied for any association. Our study is different from previous studies as most of the previous studies had used magnetic resonance imaging (MRI) brain.

\section{Methods}

This was a prospective observational study performed in the Neurology department, in association with the department of Radio-diagnosis at King George's Medical University, Lucknow, India. The institutional review board gave ethical approval. Informed written consent was taken from all the patients. Subjects were enrolled from December 2017 to February 2019 and followed up for one year. Any case with solitary intracranial parenchymal calcification and seizures was enrolled. A single round solitary calcification on CT scan was defined as calcified NCC since such lesion is considered as calcified NCC in the endemic area until proven otherwise. ${ }^{12}$ Patients with other lesions along with solitary calcification (NCC in other stages), neuroimaging suggestive of an alternative diagnosis, family history of seizures, multiple seizure types, nonepileptic events, and refusal to give consent were excluded. Seizure recurrence was defined as the recurrence of a seizure 1 week after enrollment in the study. Detailed clinical history and physical and neurological examinations were performed in all subjects. Seizure duration, semiology of seizure, postictal deficits, impairment of consciousness, occupational habits, and residence were recorded from every patient. We followed the guidelines of the International League Against Epilepsy (ILAE) for the classification of seizures. ${ }^{13}$ The socioeconomic status of the patients was classified by using Modified Kuppuswamyscale. ${ }^{14}$

Patients were separated into two groups, new-onset (Naïve) and chronic epilepsy. A new-onset seizure group was defined as a seizure onset less than 1-year duration. Biochemical investigation including liver and renal function tests were performed in all patients. Serological tests for NCC were not done as its utility is doubtful in calcified NCC. A 64-slice contrast-enhanced CT head (Philips
Brilliance $^{\mathrm{T}}{ }^{\mathrm{M}}$, Thermo Fisher Scientific, Waltham, MA, USA) was done at baseline and 9 months follow-up. The scans of $2 \mathrm{~mm}$ thickness were acquired before and after giving intravenous injection of lohexol at a dose of $1.5 \mathrm{~mL} / \mathrm{kg}$. All CT scans were analyzed by an expert radiologist who was not blinded to the study. Perilesional hypodensity on a CT scan was defined as perilesional edema. Persistence of perilesional hypodensity on follow-up scan at 9 months was defined as perilesional gliosis. ${ }^{15}$ Quantification of calcification of calcified NCC was done in terms of density, size, and volume on non-contrast CT head by using Radiant Dicom software. In this software, first, we opened the $\mathrm{CT}$ images, and then selected the calcified lesion. Using an elliptical/circular marker, we marked the calcified lesion in each slice of the CT scan, and then the area and density of calcification were calculated by the software. The slice with the maximal area and density was taken as the value for the analysis. Volume was estimated by the formula $A B C / 2$ based on the ellipsoid volume equation, where $A=$ maximum length-(in $\mathrm{cm}$ ), $B=$ width perpendicular to $A$-on the same head $\mathrm{CT}$ slice, and $\mathrm{C}=$ the number of slices multiplied by the thickness of slice. ${ }^{16}$

Electroencephalography (EEG) of 30-minute duration was done on every patient enrolled in the study. EEG recording was done with 26 electrodes (including 6 inferior temporal and 1 ECG) on the Nicolet V44 machine (Natus, USA) and the electrodes were placed following the standard 10-20 system. EEG was classified into 2 groups, normal or abnormal, and defined as abnormal if spikes, sharp waves, poly-spike or spike, and slow waves were seen. All the patients were continued on standard antiepileptic drugs if seizures were controlled. Oxcarbazepine as first-line and clobazam as the first add-on was used on patients who were not on treatment. Levetiracetam was used as a substitution for oxcarbazepine whenever needed. They were followed every 3 months and clinical features were recorded. CT scan and EEG were repeated at 9 months.

\section{Statistical analysis}

The statistical analysis was performed by using IBM SPSS Statistics Version 24.0. The categorical variables were expressed as proportions. The continuous variables were expressed as mean \pm standard deviation and Median plus Interquartile range. The categorical variables among seizure recurrence and non-recurrence groups were compared using Fisher exact test and chi-square test. The continuous variables were compared using the unpaired $t$-test. The paired continuous variables and paired categorical variables were compared using the Wilcoxon Sign Rank Sum test the McNemar tests, respectively. 
All $p$-values $<0.05$ were taken as significant. Predictors of seizure recurrence were assessed with a multivariate regression model by using binary Logistic Regression. The time to seizure recurrence analysis was performed by using the Kaplan Meier Curves/log Rank test. Furthermore, a multivariate Cox-Proportional Hazard Model was derived to predict time to seizure recurrence.

\section{Results}

The mean age of the patients was $23.33 \pm 12.81$ years (range $7-89$ years) and 71 (59.2\%) patients were males. Eighty-eight (73.3\%) patients were residents of the rural area and $80(66.67 \%)$ belonged to the lower middle class. Baseline clinical, EEG, and neuroimaging characteristics have been summarized in Supplementary Table 1. Mean density, volume, and length of calcification were $159.22 \pm 172.46 \mathrm{HU}, 0.128 \pm 0.192 \mathrm{~cm}^{3}$, and $5.33 \pm 2.23 \mathrm{~mm}$, respectively. Perilesional edema was seen in 24 cases (20\%) and contrast enhancement in 3 cases $(2.5 \%)$. The new-onset seizure was present in $22(18.3 \%), 41(34.2 \%)$ had seizure frequency more than 1 per month, and 8 had (6.7\%) abnormal EEG. Out of 120 patients 11 were lost to follow up, so finally data from 109 patients were analyzed (Supplementary Fig. 1). It showed that $35(32.11 \%)$ had seizure re-

Table 1. Clinical, EEG, and neuroimaging characteristics at 12 months $(n=109)$

\begin{tabular}{lc}
\hline Characteristic & Value \\
\hline Clinical & $35(32.11)$ \\
Seizure recurrence & \\
$\mathrm{CT}$ & \\
Density (HU) & $230.20 \pm 153.86$ \\
$\quad$ Mean $\pm \mathrm{SD}$ & $186.29(188.38)$ \\
$\quad$ Median (IQR) & \\
Volume (cm $\left.{ }^{3}\right)$ & $0.0997 \pm 0.170$ \\
$\quad$ Mean $\pm \mathrm{SD}$ & $0.0318(0.077)$ \\
$\quad$ Median (IQR) & \\
Length (mm) & $4.62 \pm 2.05$ \\
$\quad$ Mean $\pm S D$ & $4.32(2.87)$ \\
$\quad$ Median (IQR) & 0 \\
Perilesional edema & 0 \\
Perilesional gliosis & 0 \\
Contrast enhancement & \\
EEG & $4(3.3)$ \\
Abnormal & \\
\hline
\end{tabular}

$E E G$, electroencephalogram; $C T$, computed tomography; $H U$, lower density; SD, standard deviation; IQR, interquartile range.

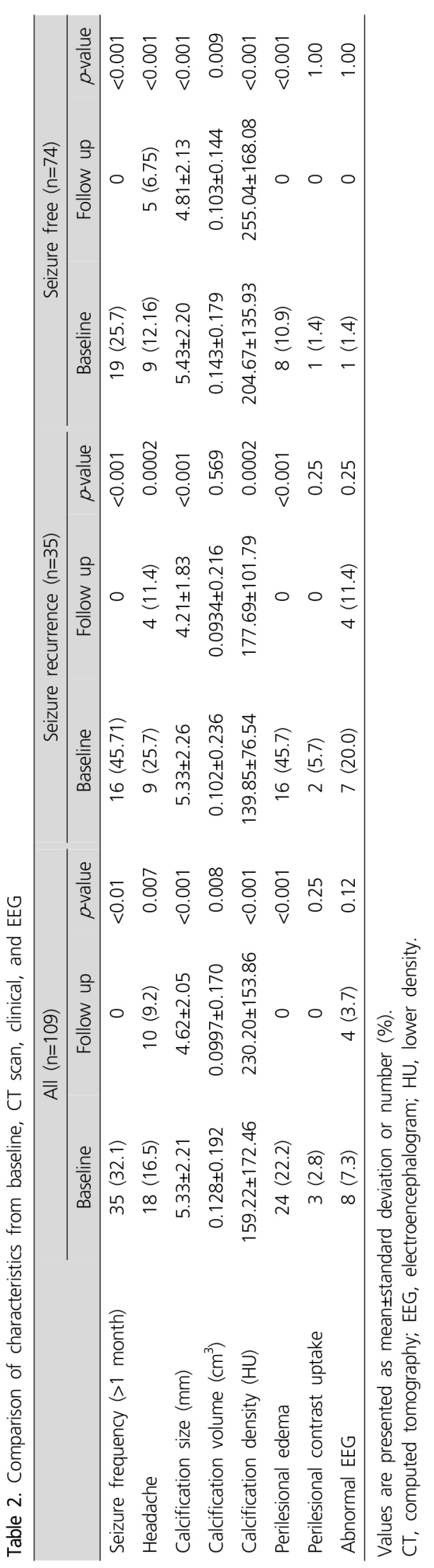


Table 3. Factors associated with the risk of seizure recurrence, binary logistic regression analysis

\begin{tabular}{lccc}
\hline Variable & B coefficient & $p$-value & Odds ratio (95\% Cl) \\
\hline Perilesional edema on CT & 1.925 & 0.001 & $6.854(2.26-20.77)$ \\
Density of lesion on CT (HU) & -0.005 & 0.036 & $0.995(0.99-1.00)$ \\
Abnormal EEG & 2.495 & 0.029 & $12.125(1.29-113.74)$ \\
\hline
\end{tabular}

$\mathrm{Cl}$, confidence interval; $\mathrm{CT}$, computed tomography; $\mathrm{HU}$, lower density; EEG, electroencephalogram.

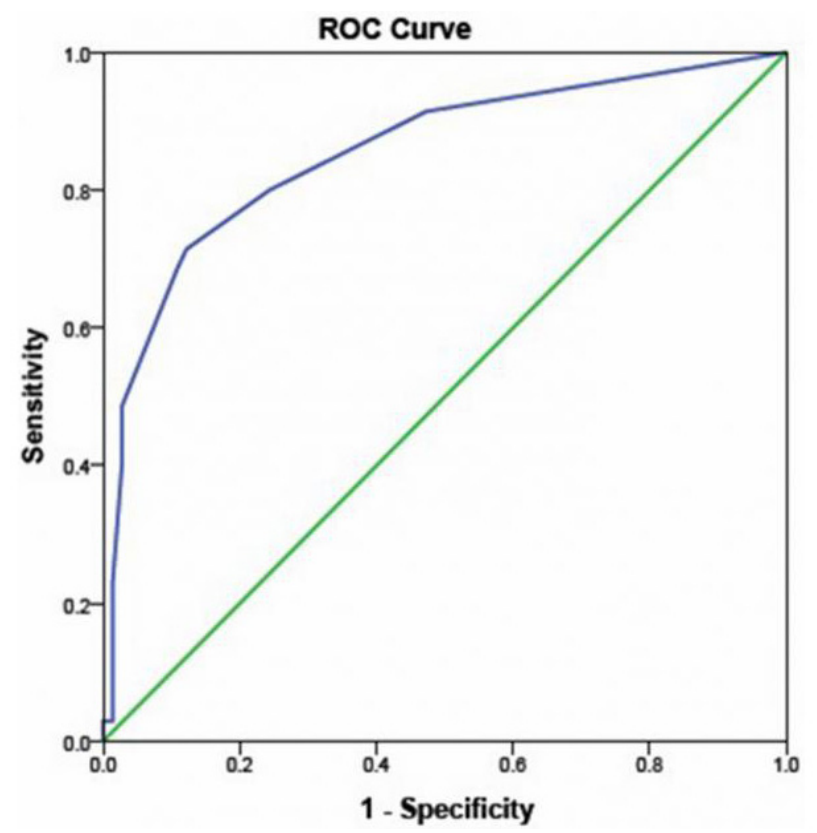

Figure 1. ROC curve derived from clinical, radiological, and electrophysiological parameters (area under the curve, 85.5\%; Homster and Lemeshow test, $p=0.58)$. ROC, receiver operating characteristic.

currence, the mean density of lesion was $230.20 \pm 153.86 \mathrm{HU}$, mean volume $0.997 \pm 0.17 \mathrm{~cm}^{3}$, mean length $4.62 \pm 2.05 \mathrm{~mm}$, and none had perilesional edema or contrast enhancement on CT scan. EEG abnormality was persistent in $4(3.7 \%)$ cases (Table 1).

On comparing from features at baseline, follow-up showed that changes in density, volume, and perilesional edema were significant. Other significant changes were the number of patients with a frequency of seizure more than 1 per month and having a headache, being 0 , and $9.2 \%$, respectively (Table 1). Two subgroups, seizure-recurrence $(n=35)$ and seizure-free $(n=74)$, had also shown similar significant changes from baseline except for calcification volume in the seizure recurrence group (Table 2). Furthermore, univariate analysis to compare all baseline parameters between seizure recurrence and seizure-free groups showed that significant parameters were seizure frequency $>1$ /month (45.7\% vs. $25.7 \%, p=0.037 ; O R, 2.06 ; 95 \%$ $\mathrm{Cl}, 1.05-5.68)$, perilesional edema ( $45.7 \%$ vs. $10.8 \%, p \leq 0.001 ; \mathrm{OR}$,

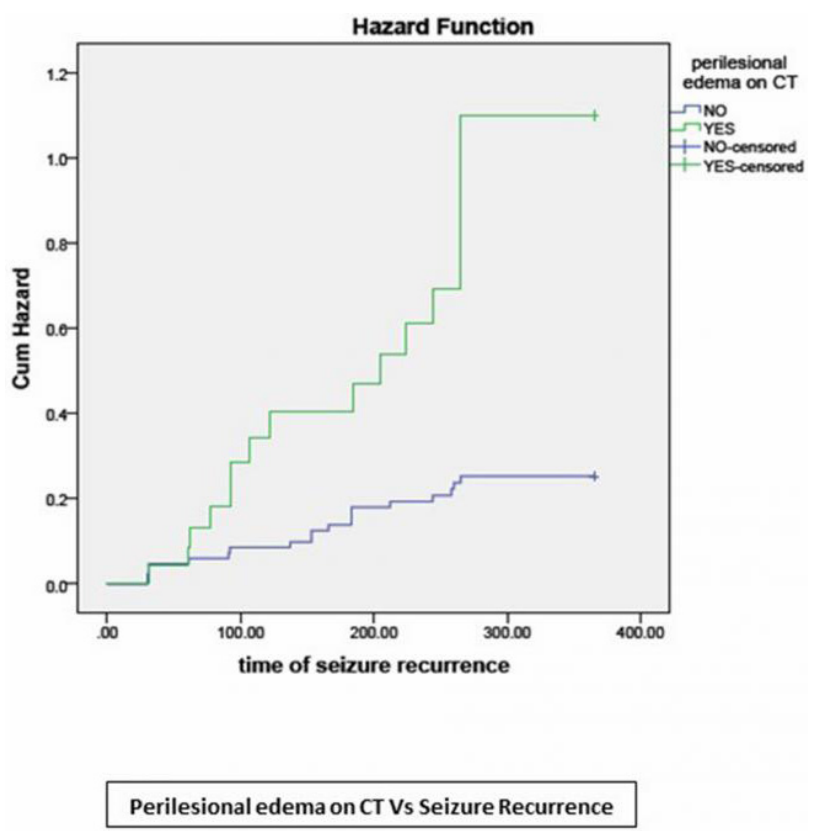

Figure 2. Seizure recurrence (cumulative hazard) with perilesional edema. $\mathrm{CT}$, computed tomography.

6.95; 95\% Cl, 2.58-18.7), lesser density of the lesion (139.85 \pm 76.54 vs. $204.67 \pm 135.9 \mathrm{HU}, p=0.009$, vector autoregression, $4.83 ; 95 \%$ $\mathrm{Cl}, 15.92-113.73$ ), and abnormal EEG (20\% vs. $1.4 \%, p \leq 0.001 ; \mathrm{OR}$, $18.25 ; 95 \% \mathrm{Cl}, 2.15-155.13$ ) (Supplementary Table 2). Binary logistic regression considering seizure recurrence as dependent variable showed that presence of perilesional edema on $\mathrm{CT}$ ( $p=0.001 ; \mathrm{OR}$, $6.854 ; 95 \% \mathrm{Cl}, 2.26-20.77)$, density of lesion on $\mathrm{CT}(\mathrm{HU})(p=0.036$; $\mathrm{OR}, 0.995 ; 95 \% \mathrm{Cl}, 0.99-1)$ and abnormal EEG $(p=0.029$; $\mathrm{OR}$, $12.125 ; 95 \% \mathrm{Cl}, 1.29-113.74)$ were the factors independently associated with seizure recurrence (Table 3).

The final model derived from these factors along with headache showed good discrimination as evident by $\mathrm{AUC}=80.9 \%(95 \% \mathrm{Cl}$, 0.72-0.90) and good calibration as evident by Homster and Lemeshow test $(p=0.743$ ) (Fig. 1). Kaplan-Meyer analysis found a significant difference in the time to event (seizure) when the patients were categorized according to the frequency of seizure $\geq 1$ episode/month (Log-rank test $p=0.036$ ) and perilesional edema on CT 
(Fig. 2, Log-rank test $p<0.001)$. The seizure recurrence rate was significantly higher in patients with frequent seizures at baseline and perilesional edema on CT Head. We have also looked at sub-lobar localization of the lesions and any association with seizure recurrence, but results were negative except that mesial frontal calcification were found to be just significant in terms of fewer chances of being associated with seizure recurrence (Supplementary Table 3).

\section{Discussion}

Neurocysticercus ends up with solitary calcification in $35-40 \%$ of cases in its natural history ${ }^{17}$ and now it is increasingly being supported with evidence about its epileptogenicity. ${ }^{7,8}$ This study found that $32.11 \%$ (35/109) cases with epilepsy and solitary calcified NCC had seizure recurrence over one year follow up. We had used stringent criteria to avoid nonepileptic attacks and other epilepsies and found that the proportions of patients with seizure recurrence are like the previous studies. ${ }^{5,18}$ Our center has been commonly seeing NCC in its various stages of natural history. It was our observation that a lower density of calcification was associated with seizure recurrence, so we wished to study this aspect prospectively. It was a novel idea and our results have shown that lower density has an association with seizure recurrence, which is being reported for the first time.

There was an increase in calcification density from baseline to final follow up which is expected as the lesion matures. The quantification parameters like volume and size had decreased at the final follow-up, showing that with the maturation calcification shrinks due to dehydration. The perilesional edema on the CT head was an independent predictor of seizure recurrence like in previous studies. ${ }^{4,5,17}$ The perilesional edema is supposed to be the transient response of the host to released antigens of parasite from the calcified lesion. ${ }^{5,7}$ We did not find any case of perilesional gliosis in our study as all the cases, who had perilesional hypodensity on CT scan, showed complete disappearance at repeat CT head at 9 months (Fig. 3). This finding contrasts with previous studies, but those were MRI-based studies and MRI is more sensitive in picking up persistent gliotic changes. ${ }^{15}$ Although we had done an MRI brain with contrast in all cases at baseline, it was not repeated at follow-up due to its cost. The baseline MRI data did not add much to what we had from the CT scan. The location of the lesion was commonest in the frontal lobe like the study by Singh et al. ${ }^{18}$, but the lobar or sub-lobar location of the lesion was not associated with any increased seizure recurrence risk in our study. This probability may be explored with a larger sample size as we had only two cases of calcified granuloma in orbitofrontal and mesial temporal regions, which are supposed to be highly epileptogenic; however, this finding might explain easily controllable seizures and infrequent drug-refractory epilepsy even in such patients.

Our findings suggest that calcified NCC may not be an inactive or inert lesion like a few other studies. ${ }^{19-21}$ This study showed that high seizure frequency at presentation was the only clinical variable that was significantly associated with the risk of seizure recurrence. This finding is like the studies wherein serial seizures and Todd's palsy was significantly associated with seizure recurrence. ${ }^{5}$ We did not observe the same high seizure frequency in patients who had seizure
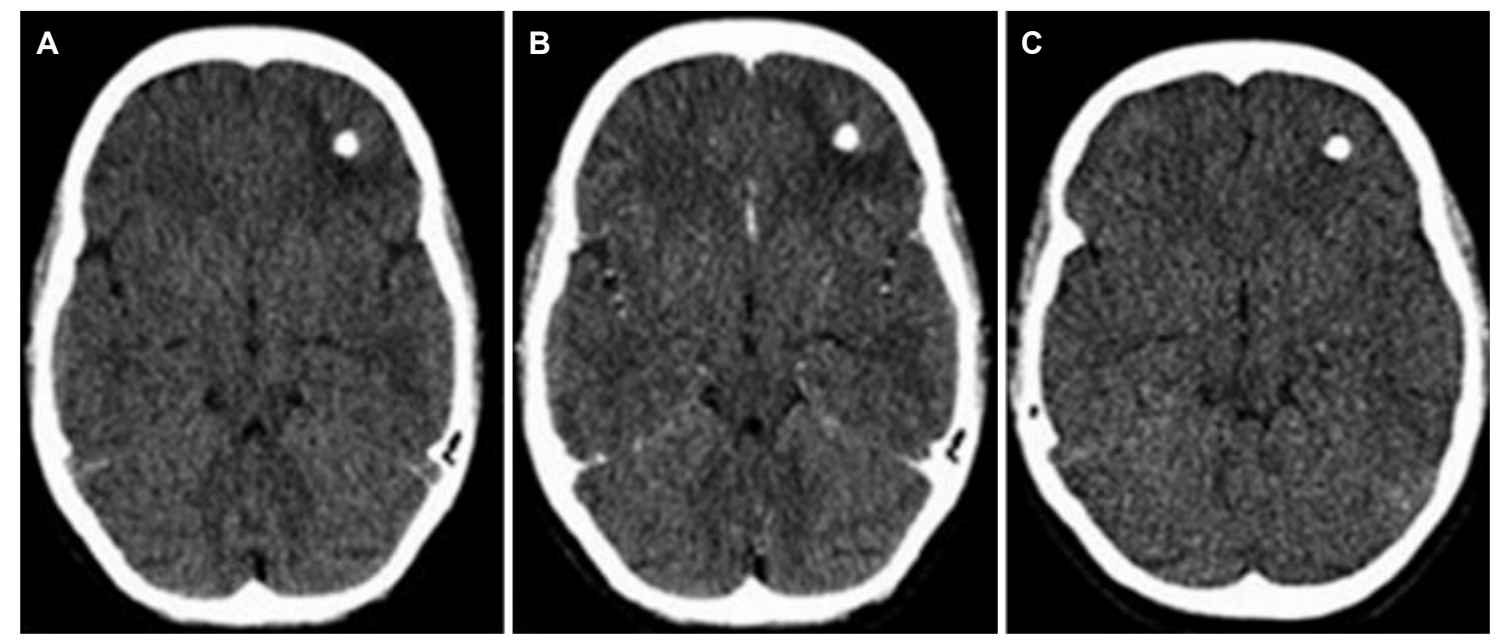

Figure 3. The computed tomography scan brain. (A) Left frontal calcified granuloma with perilesional hypodensity in plain scan. (B) Scan after contrast showing no enhancement. (C) Follow up scan at 9 months showing complete resolution of perilesional hypodensity. 
recurrence on follow-up, and this could be due to ensuring appropriate drug treatment and compliance. The EEG abnormality was found only in 8 cases (7.3\%) at baseline and 7 out of them were in the seizure recurrence group. This was again an independent predictor of seizure recurrence, which was like the previous studies., ${ }^{5,9}$ We know that the overall yield of interictal EEG is $15-50 \%$ which can further be increased by $15-35 \%$ by sleep deprivation, serial EEGs, or doing EEG within 24 hours of EEG. ${ }^{22}$ Factors like one-time EEG without sleep deprivation, irrespective of seizure timing, and episodic antigen release mediated inflammation leading to seizure development by an otherwise inert lesion could be possible reasons for the low yield of EEG in our study. Previously, another study has also shown about $8 \%$ cases with $\mathrm{EEG}$ abnormality even in patients with active lesion of cysticercosis from India. ${ }^{23}$

We studied CT characteristics of the lesion in terms of its size, volume, and density after defining the region of interest using freely available Radiant View Software. Although our method may appear crude, we found that calcification of lesser density is an important predictor of seizure recurrence, and it is a novel finding which can further be explored by using better methods. As earlier discussed, there is evidence to suggest that solitary calcified granuloma may be epileptogenic, but the reasons are unclear at present. The incomplete consolidation of calcifying granuloma or intermingling of cysticercus antigen within calcification leading to intermittent antigen release followed by transient inflammation, perilesional edema, and development of seizures have been postulated as possible mechanisms. ${ }^{24}$ Thus, immature calcification has a higher propensity to cause seizures owing to more chances of antigen-mediated inflammation. The presence of scolex within the calcified granuloma has also been found to have an increased risk of antigen-mediated inflammatory reaction and in turn seizures. ${ }^{25}$ The deleterious inflammation at consolidation or degenerating phase may cause persistent changes around calcified granuloma and pose a future risk of seizure as it is seen with perilesional gliosis. One year of follow-up is too short to comment upon seizure recurrence risk in the long term, and thus further follow-up may answer this question. Another limitation of our study was not using follow-up MRI for better assessment of perilesional gliosis. ${ }^{26}$ Moreover, we had not repeated the CT head at the time of seizure recurrence so we cannot comment about the nature of the lesion during that time. In conclusion, the lesser density of calcification and perilesional edema on CT scan of the brain, frequent seizure at presentation, and abnormal EEG are significant predictors of seizure recurrence in patients of epilepsy with solitary calcified NCC.

\section{Conflict of Interest}

The authors declare that they have no conflicts of interest.

\section{Ethical Publication Statement}

We confirm that we have read the Journal's position on issues involved in ethical publication and affirm that this report is consistent with those guidelines.

\section{Financial Support}

This study was conducted using resources of the university. No external financial support was required.

\section{Acknowledgement}

We acknowledge our institution and its research cell for the necessary financial support to conduct this study. All the authors contributed equally.

\section{References}

1. Commission on Tropical Diseases of the International League Against Epilepsy. Relationship between epilepsy and tropical diseases. Epilepsia 1994;35:89-93.

2. Garcia HH, Del Brutto OH; Cysticercosis Working Group in Peru. Neurocysticercosis: updated concepts about an old disease. Lancet Neurol 2005;4:653-61.

3. Nash TE, Del Brutto $\mathrm{OH}$, Butman JA, et al. Calcific neurocysticercosis and epileptogenesis. Neurology 2004;62:1934-8.

4. Nash TE, Pretell EJ, Lescano AG, et al. Perilesional brain oedema and seizure activity in patients with calcified neurocysticercosis: a prospective cohort and nested case-control study. Lancet Neurol 2008;7:1099-105.

5. Sharma LN, Garg RK, Verma R, Singh MK, Malhotra HS. Seizure recurrence in patients with solitary cystic granuloma or single parenchymal cerebral calcification: a comparative evaluation. Seizure 2013;22:840-5.

6. Nash TE, Mahanty S, Loeb JA, et al. Neurocysticercosis: a natural human model of epileptogenesis. Epilepsia 2015;56:177-83.

7. Lachuriya G, Garg RK, Jain A, et al. Toll-like receptor-4 polymorphisms and serum matrix metalloproteinase-9 in newly diagnosed patients with calcified neurocysticercosis and seizures. Medicine (Baltimore) 2016;95: e3288.

8. Gupta RK, Awasthi R, Rathore RK, et al. Understanding epileptogenesis in calcified neurocysticercosis with perfusion MRI. Neurology 2012;78: 618-25.

9. Del Brutto OH, Salgado P, Lama J, et al. Calcified neurocysticercosis associates with hippocampal atrophy: a population-based study. Am J Trop Med Hyg 2015;92:64-8. 
10. Cukiert A, Puglia $P$, Scapolan HB, Vilela MM, Marino Júnior $R$. Congruence of the topography of intracranial calcifications and epileptic foci. Arq Neuropsiquiatr 1994;52:289-94.

11. Rathore C, Thomas B, Kesavadas C, Abraham M, Radhakrishnan K. Calcified neurocysticercosis lesions and antiepileptic drug-resistant epilepsy: a surgically remediable syndrome? Epilepsia 2013;54:1815-22.

12. Del Brutto $\mathrm{OH}$, Rajshekhar $\mathrm{V}$, White $\mathrm{AC} \mathrm{Jr}$, et al. Proposed diagnostic criteria for neurocysticercosis. Neurology 2001;57:177-83.

13. Blume WT, Lüders HO, Mizrahi E, Tassinari C, van Emde Boas W, Engel $\mathrm{J} J \mathrm{~J}$. Glossary of descriptive terminology for ictal semiology: report of the ILAE task force on classification and terminology. Epilepsia 2001;42:1212-8.

14. Wani RT. Socioeconomic status scales modified Kuppuswamy and Udai Pareekh's scale updated for 2019. J Family Med Prim Care 2019;8:1846-9.

15. Uribe J, Olvera JE. Cysticercosis human: a clinical and pathological study of 481 autopsy patients. Patologia 1988;26:149-56.

16. Webb AJ, Ullman NL, Morgan TC, et al. Accuracy of the $A B C / 2$ score for intracerebral hemorrhage: systematic review and analysis of MISTIE, CLEAR-IVH, and CLEAR III. Stroke 2015;46:2470-6.

17. Verma A, Misra S. Outcome of short-term antiepileptic treatment in patients with solitary cerebral cysticercus granuloma. Acta Neurol Scand 2006:113;174-7.

18. Singh AK, Garg RK, Rizvi I, Malhotra HS, Kumar N, Gupta RK. Clinical and neuroimaging predictors of seizure recurrence in solitary calcified neurocysticercosis: a prospective observational study. Epilepsy Res 2017; 137:78-83.

19. Bittencourt PR, Costa AJ, Oliveira TV, Gracia CM, Gorz AM, Mazer S. Clinical, radiological and cerebrospinal fluid presentation of neurocysticercosis: a prospective study. Arq Neuropsiquiatr 1990;48:286-95.

20. Carpio A, Placencia M, Santillán F, Escobar A. A proposal for classification of neurocysticercosis. Can J Neurol Sci 1994;21:43-7.

21. Sotelo J, Guerrero V, Rubio F. Neurocysticercosis: a new classification based on active and inactive forms. A study of 753 cases. Arch Intern Med 1985; 145:442-5.

22. Wirrell EC. Prognostic significance of interictal epileptiform discharges in newly diagnosed seizure disorders. J Clin Neurophysio/ 2010;27:239-48.

23. Mehta S, Singh G. Electroclinical characteristics of new-onset seizures associated with neurocysticercosis. Neurol India 2014;62:159-63.

24. Nash TE, Bustos JA, Garcia HH; Cysticercosis Working Group in Perú. Disease centered around calcified taenia solium granuloma. Trends Parasitol 2017;33:65-73.

25. Gupta RK, Kumar R, Chawla S, Pradhan S. Demonstration of scolex within calcified cysticercus cyst: its possible role in the pathogenesis of perilesional edema. Epilepsia 2002;43:1502-8.

26. Pradhan S, Kathuria MK, Gupta RK. Perilesional gliosis and seizure outcome: a study based on magnetization transfer magnetic resonance imaging in patients with neurocysticercosis. Ann Neuro/ 2000;48:181-7. 


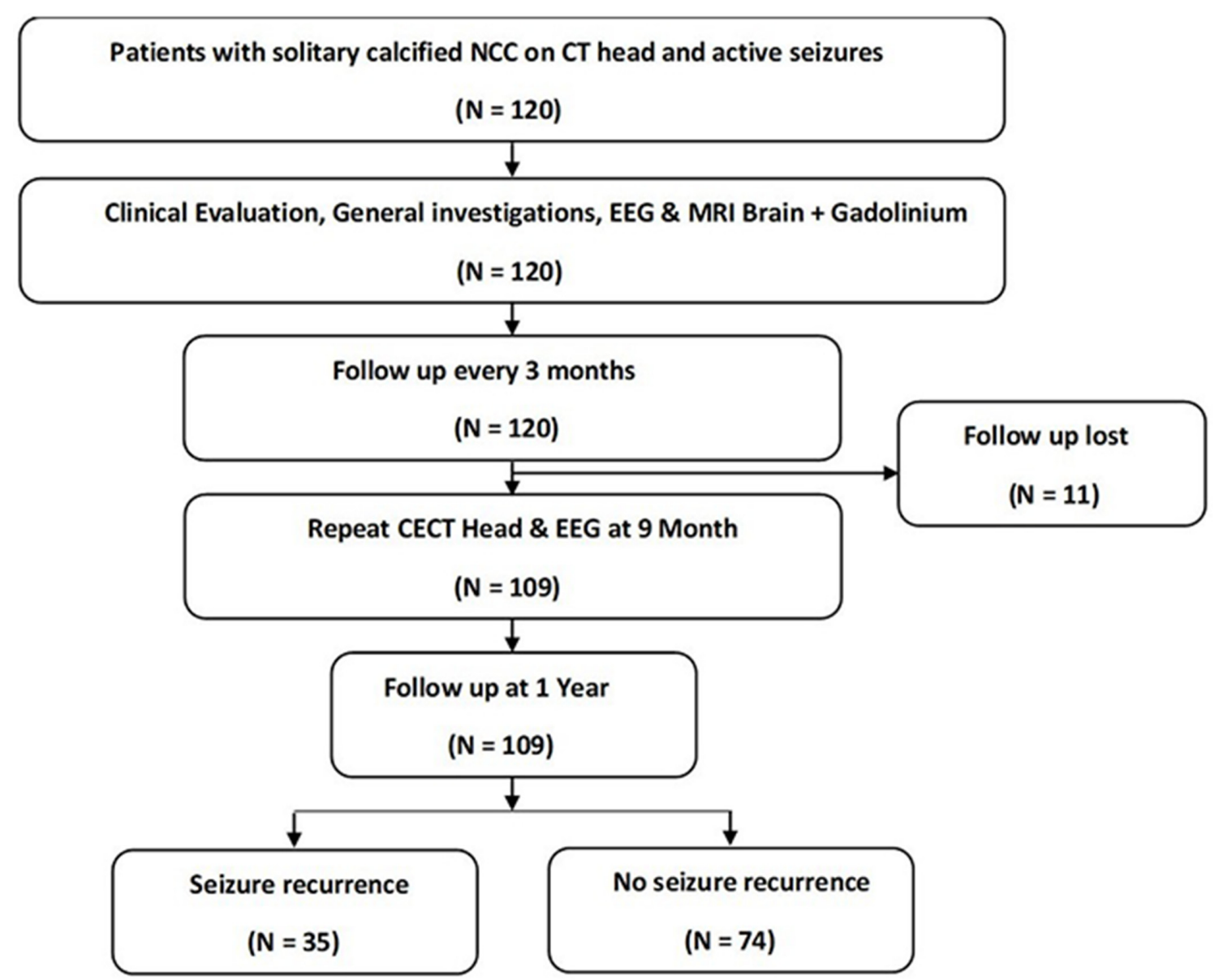

Supplementary Figure S1. Plan of study. NCC, neurocysticercosis; CT, computed tomography; EEG, electroencephalogram; MRI, magnetic resonance imaging; CECT, contrast enhanced computed tomography. 
Supplementary Table 1. Baseline characteristics ( $n=120)$

\begin{tabular}{|c|c|c|c|}
\hline Characteristic & Value & Characteristic & Value \\
\hline Age & & EEG & \\
\hline Mean \pm SD & $23.33 \pm 12.81$ & Abnormal & $8(6.7)$ \\
\hline Median (IQR) & $20(11.5)$ & CT Scan characteristics & \\
\hline Sex & & Side of lesion & \\
\hline Male & $71(59.2)$ & Left & $62(51.7)$ \\
\hline Residence & & Right & $58(48.3)$ \\
\hline Urban & $32(26.7)$ & Location of lesion & \\
\hline Rural & $88(73.3)$ & Frontal & $54(45)$ \\
\hline Socioeconomic status & & Parietal & $46(38.3)$ \\
\hline Upper & $1(0.8)$ & Temporal & $4(3.3)$ \\
\hline Upper middle & $23(19.2)$ & Occipital & $14(11.7)$ \\
\hline Lower middle & $80(66.67)$ & Caudate & $1(0.8)$ \\
\hline Upper lower & $13(10.8)$ & Hypothalamic & $1(0.8)$ \\
\hline Lower & $3(2.5)$ & Perilesional edema & $24(20)$ \\
\hline New-onset seizure & $22(18.3)$ & Contrast enhancement & $3(2.5)$ \\
\hline Seizure semiology & & Density of lesion (HU) & \\
\hline Focal & $22(18.3)$ & Mean \pm SD & $128 \pm 0.192$ \\
\hline Generalized & $62(51.7)$ & Median (IQR) & $159.22(172.46)$ \\
\hline Focal with secondary generalized & $34(28.3)$ & Volume of lesion $\left(\mathrm{cm}^{3}\right)$ & \\
\hline Undetermined & $2(1.7)$ & Mean \pm SD & $0.128 \pm 0.192$ \\
\hline Frequency of seizure & & Median (IQR) & $159.22(172.46)$ \\
\hline$<1 /$ month & $79(65.8)$ & Length of lesion (mm) & \\
\hline$\geq 1 /$ month & $41(34.2)$ & Mean \pm SD & $5.33 \pm 2.21$ \\
\hline Headache & $21(17.5)$ & Median (IQR) & 4.99 (3.04) \\
\hline Status epilepticus at presentation & $1(0.8)$ & & \\
\hline On AED at presentation & $74(61.7)$ & & \\
\hline
\end{tabular}

Values are presented as number (\%) unless otherwise indicated.

$\mathrm{SD}$, standard deviation; IQR, interquartile range; AED, antiepileptic drugs; EEG, electroencephalogram; CT, computed tomography; HU, lower density. 
Supplementary Table 2. Comparison of characteristics between seizure recurrence and seizure-free groups

\begin{tabular}{|c|c|c|c|c|}
\hline Variable $(n)$ & $\begin{array}{l}\text { Seizure-recurrence } \\
(n=35)\end{array}$ & $\begin{array}{l}\text { Seizure-free } \\
\quad(n=74)\end{array}$ & $p$-value & Odds ratio $(95 \% \mathrm{Cl})$ \\
\hline Age (years) & & & 0.83 & NA \\
\hline Mean \pm SD & $22.514 \pm 11.91$ & $23.446 \pm 13.64$ & & \\
\hline Median (IQR) & $20(11)$ & $20(10.5)$ & & \\
\hline \multicolumn{5}{|l|}{ Sex } \\
\hline Male (66) & $23(65.7)$ & $43(58.1)$ & 0.45 & $1.38(0.60-3.19)$ \\
\hline Female (43) & $12(34.33)$ & $31(41.9)$ & 0.45 & $0.72(0.31-1.67)$ \\
\hline \multicolumn{5}{|l|}{ Residence } \\
\hline Rural (80) & $27(77.1)$ & $53(71.6)$ & 0.542 & \multirow{2}{*}{$1.33(0.52-3.41)$} \\
\hline Urban (29) & $8(22.9)$ & $21(28.4)$ & 0.546 & \\
\hline \multicolumn{5}{|l|}{ Socioeconomic status } \\
\hline Upper (1) & $0(0)$ & $1(1.4)$ & 0.48 & $0.69(0.02-17.4)$ \\
\hline Upper middle (22) & $8(22.9)$ & $14(18.9)$ & 0.62 & $1.27(0.48-3.38)$ \\
\hline Lower middle (74) & $24(68.6)$ & $50(67.6)$ & 0.16 & $0.57(0.27-1.24)$ \\
\hline Upper lower (12) & $3(8.6)$ & $9(12.2)$ & 0.57 & $0.67(0.17-2.67)$ \\
\hline Lower $(0)$ & $0(0)$ & $0(0)$ & & \\
\hline \multicolumn{5}{|l|}{ Seizure onset } \\
\hline Naive (19) & $7(20)$ & $12(16.2)$ & 0.627 & $1.29(0.46-3.63)$ \\
\hline Chronic (90) & $28(80)$ & $62(83.8)$ & 0.627 & $0.77(0.28-2.18)$ \\
\hline \multicolumn{5}{|l|}{ Seizure semiology } \\
\hline Focal (21) & $7(20)$ & $14(18.9)$ & 0.89 & $1.07(0.39-2.95)$ \\
\hline Generalized (57) & $17(48.6)$ & $40(54.1)$ & 0.59 & $0.80(0.35-1.79)$ \\
\hline Secondary generalized (29) & $10(28.6)$ & $19(25.7)$ & 0.75 & $1.16(0.47-2.84)$ \\
\hline Undetermined (2) & $1(2.9)$ & $1(1.4)$ & 0.59 & $2.14(0.13-35.36)$ \\
\hline \multicolumn{5}{|l|}{ Seizure frequency } \\
\hline$\geq 1$ month (35) & $16(45.7)$ & $19(25.7)$ & 0.037 & $2.06(1.05-5.68)$ \\
\hline Headache (18) & $9(25.7)$ & $9(12.2)$ & 0.075 & $2.50(0.89-7.0)$ \\
\hline Status epilepticus (1) & $1(2.9)$ & $0(0)$ & 0.32 & $0.32(0.24-0.42)$ \\
\hline H/O medication (71) & $24(68.6)$ & $47(63.5)$ & 0.605 & $1.25(0.53-2.95)$ \\
\hline \multicolumn{5}{|l|}{ Side of lesion } \\
\hline Left (57) & $19(54.3)$ & $38(51.4)$ & 0.78 & $0.89(0.402-1.99)$ \\
\hline Right (52) & $16(45.7)$ & $36(48.6)$ & & \\
\hline \multicolumn{5}{|l|}{ Location of lesion } \\
\hline Frontal (51) & $16(45.7)$ & $35(47.3)$ & 0.91 & $1.05(0.46-2.38)$ \\
\hline Parietal (41) & $15(42.9)$ & $26(35.1)$ & 0.47 & $1.38(0.61-3.15)$ \\
\hline Temporal (4) & $1(2.9)$ & $3(4.1)$ & 0.76 & $0.69(0.07-6.94)$ \\
\hline Occipital (12) & $2(5.7)$ & $10(13.5)$ & 0.24 & $0.39(0.081-1.9)$ \\
\hline Caudate (1) & $1(2.9)$ & $0(0)$ & 0.25 & $6.56(0.26-165.2)$ \\
\hline Perilesional edema on CT (24) & $16(45.7)$ & $8(10.8)$ & $<0.01$ & $6.95(2.58-18.7)$ \\
\hline Contrast enhancement on CT (3) & $2(5.7)$ & $1(1.4)$ & 0.241 & $4.42(0.387-50.52)$ \\
\hline The density of lesion on $\mathrm{CT}(\mathrm{HU})$ & & & 0.009 (VAR $64.83,15.92$ to 113.73$)$ & NA \\
\hline Mean \pm SD & $139.85 \pm 76.54$ & $204.67 \pm 135.9$ & & \\
\hline Median (IQR) & $112.17(100.02)$ & $174.9(165.3)$ & & \\
\hline The volume of a lesion on $\mathrm{CT}\left(\mathrm{cm}^{3}\right)$ & & & 0.32 (VAR $0.041,-0.045$ to 0.123 ) & NA \\
\hline Mean \pm SD & $0.102 \pm 0.24$ & $0.143 \pm 0.18$ & & \\
\hline Median (IQR) & $0.049(0.065)$ & $0.055(0.132)$ & & \\
\hline Length of a lesion on CT (mm) & & & 0.82 (VAR 0.1, -0.81 to 1.003 ) & NA \\
\hline Mean \pm SD & $5.33 \pm 2.26$ & $5.43 \pm 2.20$ & & \\
\hline Median (IQR) & $4.64(2.50)$ & $5.27(3.42)$ & & \\
\hline EEG abnormal (8) & $7(20)$ & $1(1.4)$ & $<0.01$ & $18.25(2.15-155.13)$ \\
\hline
\end{tabular}

Values are presented as number (\%) unless otherwise indicated.

$\mathrm{Cl}$, confidence interval; SD, standard deviation; NA, not available; IQR, interquartile range; $\mathrm{H} / \mathrm{O}$, history of; $\mathrm{CT}$, computed tomography; $\mathrm{HU}$, lower density; VAR, vector autoregression; EEG, electroencephalogram. 
Supplementary Table 3. Seizure recurrence risk with sub lobar localization of calcification

\begin{tabular}{|c|c|c|c|c|}
\hline Location & $\begin{array}{l}\text { Seizure recurrence } \\
\qquad(n=35)\end{array}$ & $\begin{array}{l}\text { No recurrence } \\
\quad(n=74)\end{array}$ & $p$-value & Odds ratio $(95 \% \mathrm{Cl})$ \\
\hline \multicolumn{5}{|l|}{ Frontal } \\
\hline Orbitofrontal & 0 & 0 & & \\
\hline Polar & 0 & $1(1.4)$ & 0.8 & $0.69(0.027-17.6)$ \\
\hline Dorsolateral & $5(14.3)$ & $3(4.1)$ & 0.07 & $3.9(0.88-17.5)$ \\
\hline Ventrolateral & $7(20)$ & $14(18.9)$ & 0.84 & $1.1(0.4-3.06)$ \\
\hline Superolateral & $3(8.5)$ & $2(2.7)$ & 0.18 & $3.4(0.54-21.48)$ \\
\hline Mesial & $1(2.8)$ & $14(18.9)$ & 0.05 & $0.12(0.015-1.00)$ \\
\hline Opercular & 0 & $1(1.4)$ & 0.8 & $0.69(0.027-17.6)$ \\
\hline \multicolumn{5}{|l|}{ Parietal } \\
\hline Anterolateral & $2(5.7)$ & $6(8.11)$ & 0.65 & $0.68(0.13-3.5)$ \\
\hline Posterolateral & $5(14.27)$ & $14(18.91)$ & 0.71 & $0.71(0.25-2.17)$ \\
\hline Mesial & $6(17.14)$ & $6(8.10)$ & 0.15 & $2.42(0.72-8.71)$ \\
\hline Opercular & $2(5.7)$ & 0 & 0.12 & $11.1(0.51-238.02)$ \\
\hline \multicolumn{5}{|l|}{ Temporal } \\
\hline Anterolateral & 0 & $1(1.4)$ & 0.8 & $0.69(0.027-17.6)$ \\
\hline Posterolateral & 0 & $2(2.7)$ & 0.56 & $0.40(0.019-8.73)$ \\
\hline Mesial & $1(5.7)$ & 0 & 0.26 & $6.47(0.25-163.12)$ \\
\hline Basal & 0 & 0 & & \\
\hline Opercular & 0 & 0 & & \\
\hline \multicolumn{5}{|l|}{ Occipital } \\
\hline Anterolateral & $1(2.8)$ & 0 & 0.25 & $6.4(0.25-163.12)$ \\
\hline Posterolateral & 0 & $1(1.4)$ & 0.8 & $0.69(0.027-17.6)$ \\
\hline Mesial & $1(2.8)$ & 9 (12.16) & 0.14 & $0.21(0.25-1.74)$ \\
\hline
\end{tabular}

Values are presented as number (\%). 